UT-878

\title{
Remarks on Shannon's Statistical Inference and the Second Law in Quantum Statistical Mechanics
}

\author{
Kazuo Fujikawa \\ Department of Physics, University of Tokyo \\ Bunkyo-ku,Tokyo 113,Japan
}

\begin{abstract}
We comment on a formulation of quantum statistical mechanics, which incorporates the statistical inference of Shannon. Our basic idea is to distinguish the dynamical entropy of von Neumann, $H=-k \operatorname{Tr} \hat{\rho} \ln \hat{\rho}$, in terms of the density matrix $\hat{\rho}(t)$, and the statistical amount of uncertainty of Shannon, $S=-k \sum_{n} p_{n} \ln p_{n}$, with $p_{n}=\langle n|\hat{\rho}| n\rangle$ in the representation where the total energy and particle numbers are diagonal. These quantities satisfy the inequality $S \geq H$. We propose to interprete Shannon's statistical inference as specifying the initial conditions of the system in terms of $p_{n}$. A definition of macroscopic observables which are characterized by intrinsic time scales is given, and a quantum mechanical condition on the system, which ensures equilibrium, is discussed on the basis of time averaging. An interesting analogy of the change of entroy with the running coupling in renormalization group is noted. A salient feature of our approach is that the distinction between statistical aspects and dynamical aspects of quantum statistical mechanics is very transparent.
\end{abstract}

\section{Introduction}

$A$ formulation of statistical mechanics on the basis of Shannon's information theory ${ }^{1)}$ has been proposed by Jaynes in 1957.2) This formulation utilizes the least biased statistical inference about a physical system on the basis of a limited amount of information available.

In the present note we discuss quantum statistical mechanics, ${ }^{3-9)}$ which incorporates Jaynes's proposal, and we examine what kind of picture appears if one distinguishes the statistical aspects and dynamical aspects of quantum statistical mechanics to the maximum extent. We work exclusively on quantum statistcal mechanics, though in the course of our discussion we comment on the recent progress in the Boltzmann approach to classical statistical mechanics.$^{10-12)}$

We would like to briefly summarize the basic aspects of our analysis. We examine a quantum mechanical mixed state, which is slightly away from thermal equilibrium, in the representation where the total energy and particle numbers are diagonal. The average value of any macroscopic observable $\hat{O}$ is given by $\langle\hat{O}(t)\rangle=\operatorname{Tr} \hat{O} \hat{\rho}(t)$. For the system slightly away from equilibrium, $\langle\hat{O}(t)\rangle$ is time dependent in general, and thus the density 
matrix $\hat{\rho}(t)$ is time dependent. Following von Neumann, we introduce the (dynamical) entropy defined by

$$
H=-k \operatorname{Tr} \hat{\rho} \ln \hat{\rho}
$$

which is a generalization of Gibbs entropy to a quantum system. It is well known that $H$ thus defined is constant in time.

The basic observation in this note is that we can define another quantity

$$
S=-k \sum_{n} p_{n} \ln p_{n}
$$

in terms of $p_{n} \equiv\langle n|\hat{\rho}| n\rangle$ in the representation where the total energy and particle numbers are diagonal. By definiton we have $p_{n}(t)=p_{n}(0)$ and thus $S$ is also constant in time. We propose to identify $S$ thus defined, which can be regarded as basically statistical quantity, as the amount of uncertainty of Shannon which was introduced into statistical mechanics by Jaynes. ${ }^{2)}$ Note that $H$ in (1.1) agrees with $S$ in (1.2) only when $\hat{\rho}$ is diagonalizable simultaneously with the total Hamiltonian and the total particle number operator. This clear distinction between $H$ in (1.1) and $S$ in (1.2), to our knowledge, has not been made in the past. ${ }^{13)}$

In the present formulation of quantum statistical mechanics, we regard the least biased estimate on the basis of limited amount of information discussed by Jaynes as a least biased estimate of initial conditions on the diagonal elements $p_{n}$. Note that the diagonal elements of $\hat{\rho}(t)$ are constant in time and thus they cannot approach equilibrium values by any dynamical motion but by statistical inference. (Our initial conditions are thus final conditions also.) The initial state is thus specified by $\left\{p_{n}\right\}$ and a set of macroscopic observables other than the total energy and particle number. From the days of Boltzmann, it is well known that the second law of thermodynamics is mechanically represented only by means of assumptions regarding initial conditions, ${ }^{12)}$ or by choosing the typical states in the classical Boltzmann analysis. ${ }^{11)}$ In quantum statistical mechanics, as formulated here, it is clear that Shannon's statistical inference does not precede physical dynamics.

As for the main problem of quantum statistical mechanics as to what kind of dynamical properties of the system ensure the approach to eventual thermal equilibrium, we propose to analyze a set of macroscopic observables, each of which is characterized by an intrinsic time scale $\tau$. As for the entropy law, some form of coarse graining is necessary not only in the Gibbs approach to quantum statistical approach ${ }^{8)}$ but also in the Boltzmann approach

to classical statistical mechanics. ${ }^{10)}$ In our approach it turns out to be more convenient to take a coarse graining in the "time direction" or a suitable time averaging. ${ }^{14,9)}$ The entropy law of Clausius in the present formulation is expressed as the approach of the macroscopic observables of the system on a suitable time average to those of the almost equilibrium state, whose physical entropy is estimated by the maximum value of Shannon's $S$.

\section{Shannon's Least Biased Inference}

We consider the variable $x$ which takes the $n$ values $\left\{x_{1}, x_{2}, \ldots, x_{n}\right\}$ and define the probability $p_{i}$ for the variable $x$ to assume the value $x_{i}$. The non-negative probability $p_{i}$ is 
constrained by the condition

$$
\sum_{i=1}^{n} p_{i}=1
$$

which means that the total probability is unity. We also consider a smooth function $f(x)$ of the variable $x$, such as $f(x)=x$.

We then ask what we can say about the set of probabilities $\left\{p_{1}, p_{2}, \ldots, p_{n}\right\}$, if only available information is the average value $\langle f>$ of $f(x)$ defined by

$$
<f>\equiv \sum_{i=1}^{n} p_{i} f\left(x_{i}\right) \text {. }
$$

Clearly it is impossible to determine all $p_{i}$ uniquely for a large value of $n$ since we know only $<f>$.

Shannon introduced the notion of amount of uncertainty $S\left(p_{1}, p_{2}, \ldots, p_{n}\right)$ for the set of variables $\left\{p_{1}, p_{2}, \ldots, p_{n}\right\}$, and he proposed to determine each $p_{i}$ by allowing the maximum amount of uncertainty, or equivalently, the least bias for the chosen solution of $\left\{p_{1}, p_{2}, \ldots, p_{n}\right\}$. On the basis of a composition law, Shannon derived the amount of uncertainty $^{1)}$ ( see also Appendix in ref. 2)

$$
S\left(p_{1}, p_{2}, \ldots, p_{n}\right)=-k \sum_{i=1}^{n} p_{i} \ln p_{i}
$$

with a positive constant $k$.

We now apply the above theory of inference to statistical mechanics. Consider a closed system for which one knows that the total energy is confined within a small range

$$
E-\frac{1}{2} \Delta E \leq E_{n} \leq E+\frac{1}{2} \Delta E
$$

with the constraint

$$
\sum_{n} E_{n} p_{n}=E
$$

for sufficiently small $\Delta E$ (with a fixed particle number $N$ and a fixed volume). The maximum of the Shannon's amount of uncertainty then gives rise to the probability for $E_{n}$

$$
\begin{aligned}
& p_{n}=\exp \left[-\beta E_{n}\right] / Z, \\
& Z=\sum_{E-\frac{1}{2} \Delta E \leq E_{n} \leq E+\frac{1}{2} \Delta E} \exp \left[-\beta E_{n}\right] .
\end{aligned}
$$

The parameter $\beta=\beta(E)$, which is introduced as a multiplier, is defined by

$$
E=-\frac{\partial \ln Z}{\partial \beta}
$$

This formulation, which exhibits the temperature explicitly, is more convenient than the conventional formulation in microcanonical ensemble. ${ }^{15)}$ This formulation, if one assumes 
static equilibrium, is reduced to the microcanonical ensemble in the limit of small $\Delta E$ : The equal a priori probabilities are obtained as

$$
p_{n} \simeq \frac{\exp [-\beta E]}{\Delta W(E, N) \exp [-\beta E]}=\frac{1}{\Delta W(E, N)}
$$

and the thermodynamic relation

$$
\begin{aligned}
F & =-\frac{1}{\beta} \ln Z \\
& \simeq-\frac{1}{\beta} \ln \{\Delta W(E, N) \exp [-\beta E]\} \\
& =E-\frac{1}{\beta} S / k=E-T S .
\end{aligned}
$$

Here we used the Boltzmann entropy

$$
S=k \ln \Delta W(E, N) \simeq-k \sum_{n} p_{n} \ln p_{n}
$$

where $\Delta W(E, N)$ stands for the number of quantum states within the above energy range. Our determination of the time independent quantities $p_{n}$ is thus consistent with the microcanonical ensemble for sufficiently small $\Delta E$.

In the next section, we discuss a formulation of quantum statistical mechanics which incorporates the above statistical inference, and yet a logically consistent formulation in the sense that statistical inference does not precede dynamical time development. Note that we rely on the statistical inference since we deal with an enormous number of states $E_{n}$ in (2.4).

\section{Statistical Inference and Quantum Statistical Me- chanics}

We discuss how to describe near-equilibrium states and their approach to thermal equilibrium in a framework which incorporates the least biased statistical inference. We assume that we analyze a physical system which is completely characterized by its total energy and particle number, if the thermal equilibrium should be realized for a fixed volume. In the representation where the total energy and the particle number are diagonal, we have the density matrix ${ }^{16)} \hat{\rho}(t)$ which satisfies ${ }^{8)}$

$$
\begin{aligned}
& \operatorname{Tr} \hat{\rho}(t)=\sum_{n} \hat{\rho}(t)_{n n}=\sum_{n} p_{n}=1, \\
& \operatorname{Tr} \hat{\rho} \hat{\mathcal{H}}=\sum_{n} E_{n} p_{n}=\langle E\rangle, \\
& \operatorname{Tr} \hat{\rho} \hat{\nu}=\sum_{n} \nu_{n} p_{n}=\langle N\rangle .
\end{aligned}
$$


We here assume for simplicity the presence of only one kind of particles. We first note that $p_{n}$ is time independent $p_{n}=\langle n|\hat{\rho}(t)| n\rangle=\left\langle n\left|e^{-i \hat{\mathcal{H}} t / \hbar} \hat{\rho}(0) e^{i \hat{\mathcal{H}} t / \hbar}\right| n\right\rangle=\langle n|\hat{\rho}(0)| n\rangle$, since the total Hamiltonian is diagonal in the present representation. We assume that either none of the energy levels are degenerate, or if some of them are degenerate, the density matrix $\hat{\rho}(t)$ is diagonalized by a (constant) unitary transformation beforehand in each sector which contains the degenerate energy levels. Consequently, all the possible off-diagonal elements of the density matrix $\hat{\rho}(t)$ are time dependent. Our proposal is to define the Shannon's amount of uncertainty, which is based on the information available, by

$$
S=-k \sum_{n} p_{n} \ln p_{n}
$$

This $S$, which carries no characteristic properties of quantum theory, may be assigned a purely statistical meaning, and it is time independent.

The dynamical entropy of von Neumann

$$
H=-k \operatorname{Tr} \hat{\rho} \ln \hat{\rho}
$$

which is a quantum generalization of Gibbs entropy, is also time independent since the time development of $\hat{\rho}$ is a unitary transformation. In ref. 9 (and also in ref. 5), this entropy $H$, which in principle contains the effects of quantum coherence, is called the information entropy. In this paper we stick to the classical notion of information and thus to the amount of uncertainty defined in (3.2). The advantage of choosing (3.2) as the amount of uncertainty becomes clear later. The average value of any operator in the Schrödinger representation is defined by

$$
\langle\hat{O}(t)\rangle=\operatorname{Tr} \hat{\rho}(t) \hat{O}(0)
$$

In the framework of Shannon's least biased statistical inference used by Jaynes, the maximum value of the amount of uncertainty, which is identified with $S$ in (3.2) in the present formulation, is considered with the constraints (3.1). For a closed system we are considering, the constraints are actually replaced by the conditions (2.4) and (2.5) in Section 2. One then obtains the standard result (2.6) for the constant diagonal elements of $\hat{\rho}(t)$

$$
\begin{aligned}
& p_{n}=\exp \left[-\beta(E) E_{n}\right] / Z, \\
& Z=\sum_{E-\frac{1}{2} \Delta E \leq E_{n} \leq E+\frac{1}{2} \Delta E} \exp \left[-\beta(E) E_{n}\right]
\end{aligned}
$$

but the time dependent off-diagonal elements of $\hat{\rho}(t)$ are left completely unspecified, namely, we remain maximally noncommittal with regard to missing information. ${ }^{17)}$

On the other hand, the maximum of the von Neumann entropy $H$ with conditions (2.4) and (2.5) would give rise to ${ }^{6,7,8)}$

$$
\begin{aligned}
\langle n|\hat{\rho}(t)| n\rangle & =\exp \left[-\beta(E) E_{n}\right] / Z \\
\langle n|\hat{\rho}(t)| m\rangle & =0, \quad n \neq m .
\end{aligned}
$$


This density matrix $\hat{\rho}$ is completely diagonal; in other words, if one imposes the maximum condition on the von Neumann entropy, we arrive at the conventional microcanonical ensemble without any freedom of time development. In fact, the conventional analysis of statistical mechanics utilizes this property of the entropy $H$ and attempts to prove the Boltzmann's H-theorem for (a coarse grained form of) $H$ in (3.3) as an indicator of the general tendency toward thermal equilibrium. ${ }^{8,9,18)}$ In contrast, we here attempt to characterize the approach to thermal equilibrium from a different perspective. Also, we will later suggest that the dynamical density matrix does not approach the static form (3.6) even in thermal equilibrium, since the oscillations with microscopic time scales, which should be described by the dynamical density matrix, always exist in the system.

For the general situation, the maximum uncertainty inference of Shannon as formulated here does not specify the density matrix completely, and the best estimate of the average of a general macroscopic operator $\hat{O}$ is

$$
\langle\hat{O}\rangle_{0} \equiv \sum_{n} p_{n} \hat{O}(0)_{n n}=\sum_{n} \hat{O}(0)_{n n} \exp \left[-\beta(E) E_{n}\right] / Z
$$

where $p_{n}$ is defined in (3.5) and the sum is taken over the available eigenstates in the representation where the total Hamiltonian and particle number are diagonal. This quantity is time independent by definition and agrees with the conventional average in thermal equilibrium. We will later show that, only after a suitable time averaging, the true average (3.4) is well approximated by the conventional thermal average (3.7) if the system satisfies certain dynamical properties. It is clear that the least biased inference of Shannon, as formulated here, is purely statistical and does not provide any information about dynamical time development.

We next note an inequality between $S$ and $H$ (see also refs. 5 and 19)

$$
H \leq S
$$

This relation is shown by using the standard technique of the statistical mechanics ${ }^{20)}$. This inequality, $H \leq S$, valid for any $\hat{\rho}(t)$ suggests that we can impose the maximum amount of uncertainty condition on $S$ without any dynamical constraint on $H$. (In contrast, if one should use (3.3) as Shannon's amount of uncertainty, the statistical inference would precede physical dynamics, since the statistical inference would then determine the time dependent off-diagonal elements of $\hat{\rho}(t)$ as well.) Besides, $H \leq S$ shows that we start with an initial state with smaller entropy. ${ }^{12)}$

In analogy with the definition of a quantum state in terms of a complete set of commuting hermitian operators, ${ }^{22)}$ we assume that our density matrix $\hat{\rho}(t)$, which is a generalization of the Schrödinger wave function, is well specified by a set of macroscopic observables $\{\hat{O}\}$, and total energy and particle number; in the present case, the operators $\{\hat{O}\}$ do not commute with the total Hamiltonian by our assumption. As in classical Boltzmann statistical mechanics, ${ }^{11)}$ where one works exclusively on macroscopic variables, one may define macroscopic observables $\hat{O}_{\tau}$ in the present framework by the condition

$$
\operatorname{Tr} \hat{\rho}(t) \hat{O}_{\tau}=\frac{1}{\tau} \int_{t}^{t+\tau} d t \operatorname{Tr} \hat{\rho}(t) \hat{O}_{\tau}
$$


This condition is written in full detail as

$$
\begin{aligned}
\left\langle\hat{O}_{\tau}(t)\right\rangle & =\sum_{m, n} \rho_{m n}(0)\left\langle n\left|\hat{O}_{\tau}\right| m\right\rangle \exp \left[i\left(E_{n}-E_{m}\right) t / \hbar\right] \\
& =\frac{1}{\tau} \int_{t}^{t+\tau} d t \sum_{m, n} \rho_{m n}(0)\left\langle n\left|\hat{O}_{\tau}\right| m\right\rangle \exp \left[i\left(E_{n}-E_{m}\right) t / \hbar\right]
\end{aligned}
$$

and thus the macroscopic observables are not sensitive to the microscopic time (shorter than $\tau$ ) dependence of the density matrix. ${ }^{23)}$ Each macroscopic observable in our definition is labeled by a characteristic time scale $\tau$, which may in general depend on the temperature contained in the diagonal components $\left\{p_{n}\right\}$ of the density matrix. ${ }^{24)}$ An operator with large $\tau$ gives a macroscopic observable which agrees with our intuitive understanding: For example, $\tau=\infty$ for the total energy or particle number.

We then define the non-equilibrium state operationally by the relation

$$
\begin{aligned}
\left\langle\Delta \hat{O}_{\tau}(t)\right\rangle & \equiv \operatorname{Tr} \hat{\rho}(t)\left[\hat{O}_{\tau}-\sum_{n} p_{n}<n\left|\hat{O}_{\tau}\right| n>\right] \\
& =\operatorname{Tr} \hat{\rho}(t) \hat{O}_{\tau}-\sum_{n} p_{n}<n\left|\hat{O}_{\tau}\right| n>\neq 0
\end{aligned}
$$

for some macroscopic observables $\hat{O}_{\tau}$ other than the total energy and particle number. Here $p_{n}$ is defined in (3.5). If we do not find any sensible macroscopic observable $\hat{O}_{\tau}$ which satisfies the above relation ( after a suitable time averaging described later ), the system is in thermal equilibrium.

Our system described by the density matrix $\hat{\rho}(t)$ then develops with time following Schrödinger equation with a fixed value of the von Neumann entropy $H$.

\section{Second Law in Quantum Statistical Mechanics}

Our next task is to specify what kind of dynamical properties of a many particle system ensure that the system with initial conditions defined by our statistical inference will in the long run approach the almost equilibrium state. We first note that the time average of our $\hat{\rho}(t)$ over a sufficiently long period approaches arbitrarily close to the equilibrium $\hat{\rho}_{0}$ with diagonal elements $p_{n}$ in (3.5). In this sense, our system by its construction satisfies the Boltzmann's ergodic postulate; the time average behavior of a system is the same as its equilibrium behavior. Namely a suitable time averaging of (3.11) gives rise to

$$
\left\langle\overline{\Delta \hat{O}_{\tau}}\right\rangle \simeq 0
$$

which is the (necessary) condition for equilibrium.

We need to sharpen the time averaged behavior (4.1) to be a sufficient dynamical condition for equilibrium. We first recall a quantum version of Poincare's recurrence theorem. ${ }^{25)}$ The theorem states that observables in a (finite) many particle system with discrete energy spectrum are almost periodic, namely, after a suitable time lapse the system comes back to arbitrarily close to the original configuration. This means that the 
system has a rather well defined dynamical property and we here deal with those finite systems. ${ }^{26)}$ We however assume that the recurrence time for a many particle system is sufficiently long by the time scale of our laboratory. The reccurence theorem provides a partial justification for the independent specification of diagonal elements of $\hat{\rho}$ in (3.5) and the non-diagonal elements in (3.11), since an apparently thermal equilibrium state, which is related to $(3.5)$, can come back to any original starting configuration.

To ensure the thermal equilibrium, we need to avoid the persistent synchronized collective oscillation even if the averaged behavior (4.1) is satisfied. We expect that the probability for a great number of oscillators in $\hat{\rho}(t)$ to synchronize persistently is negligibly small for a system of a many particle system. In the generic situation, our system is expected to give a negligible time correlation between $\left\langle\Delta \hat{O}_{\tau}(t)\right\rangle$ for a large time difference $\left|t_{1}-t_{2}\right|$, which is however very small compared to the recurrence time.

The averaged behavior (4.1) is now sharpened to be a stronger dynamical postulate as ( see also (3.11))

$$
\begin{aligned}
\left|\left\langle\overline{\Delta \hat{O}_{\tau}}\right\rangle\left(t, \Delta t_{c}\right)\right| & \equiv\left|\frac{1}{\Delta t_{c}} \int_{t}^{t+\Delta t_{c}} d t\left\langle\Delta \hat{O}_{\tau}(t)\right\rangle\right| \\
& =\left|\frac{1}{\Delta t_{c}} \int_{t}^{t+\Delta t_{c}} d t \operatorname{Tr} \hat{\rho}(t) \hat{O}_{\tau}-\sum_{n} p_{n}<n\right| \hat{O}_{\tau}|n\rangle \mid \\
& \ll\left|\left\langle\hat{O}_{\tau}\right\rangle_{0}\right|
\end{aligned}
$$

for any macroscopic observable $\hat{O}_{\tau}$ and a fixed finite $\Delta t_{c} ;\left\langle\hat{O}_{\tau}\right\rangle_{0}=\sum_{n} p_{n}\left\langle n\left|\hat{O}_{\tau}\right| n\right\rangle \mid$ is defined in (3.7) with $p_{n}$ in (3.5). For $\Delta t_{c}=\infty$, the left-hand side of this relation vanishes by our construction. We impose this condition for a finite $\Delta t_{c}$ and assume that $\left\langle\overline{\Delta \hat{O}_{\tau}}\right\rangle\left(t, \Delta t_{c}\right)$ is not sensitive to the absolute value of $t$ and a small variation of $\Delta t_{c}$. Physically, this condition means the existence of a well-defined relaxation time for a set of macroscopic observables. The actual magnitude of $\Delta t_{c}$, which is expected to be microscopically quite long and of the order of macroscopic time scale, will generally depend on the specific system we are analyzing. In terms of the parameter $\tau$ in (3.9), we expect (for an operator with a finite $\tau$ )

$$
\tau<\Delta t_{c}<\infty
$$

To observe the relaxation in terms of the macroscopic observables $\hat{O}_{\tau}$, we need to have $\tau<\Delta t_{c}$.

Note that the condition (4.2) does not contradict the reccurence theorem: ${ }^{25)}$ In practice, after the initial relaxation, it might be that one can take $\Delta t_{c} \sim \tau$, namely, one cannot recognize the sizable deviation from thermal equilibrium by the time resolution of macroscopic observables. However, if the reccurence occurs, one need to wait for the time $\sim \Delta t_{c}$ for the system to relax again.

Since

$$
\left\langle\hat{O}_{\tau}(t)\right\rangle=\sum_{m, n} \rho_{m n}(0)\left\langle n\left|\hat{O}_{\tau}\right| m\right\rangle \exp \left[i\left(E_{n}-E_{m}\right) t / \hbar\right]
$$

we have only the near diagonal components after the above time averaging (4.2); namely, only the terms with

$$
\left|E_{n}-E_{m}\right| \leq 2 \pi \hbar / \Delta t_{c}
$$


survive the time averaging. Note that $2 \pi \hbar / \Delta t_{c}$ is very small compared to $\Delta E$ in (3.5) for a many particle system we are interested in. ${ }^{27)}$ Also the real time-independent diagonal components are subtracted in $\left\langle\Delta \hat{O}_{\tau}(t)\right\rangle$. In the remaining terms we have a sum of complex amplitudes $\rho_{m n}(0)\left\langle n\left|\hat{O}_{\tau}\right| m\right\rangle$ with nearly equal frequencies $\left(E_{n}-E_{m}\right) / \hbar$ : Our condition (4.2) is that the sum of oscillating quantities are either absent or destructively interfere

$$
\left|\sum_{0<\left|E_{n}-E_{m}\right| \leq 2 \pi \hbar / \Delta t_{c}} \rho_{m n}(0)\left\langle n\left|\hat{O}_{\tau}\right| m\right\rangle \exp \left[i\left(E_{n}-E_{m}\right) t / \hbar\right]\right| \ll\left|\left\langle\hat{O}_{\tau}\right\rangle_{0}\right|
$$

for a general value of $t$.

As a concrete example of our analysis, we illustrate the problem of a gas confined in the left-half of a box and then removing the partition, although this probelm corresponds to the case of far from equilibrium. ${ }^{28)}$ We make a statistical inference to fix $p_{n}$ on the basis of the information about all the possible energy spectra of the entire box, the average energy and particle number. The macroscopic observable $\hat{O}_{\tau}(\vec{x})$ may be chosen as the particle number density (in a suitably smeared sense) inside the entire box. We define $\left\langle\hat{O}_{\tau}\right\rangle_{0}(\vec{x})$ by using the result of the above inference and (3.7). The observable

$$
\operatorname{Tr} \hat{\rho}(t) \Delta \hat{O}_{\tau}(\vec{x})=\operatorname{Tr} \hat{\rho}(t) \hat{O}_{\tau}(\vec{x})-\left\langle\hat{O}_{\tau}\right\rangle_{0}(\vec{x}),
$$

which has a positive peak in the left-half of the box and a negative peak in the right-half of the box at $t=0$, is described by choosing the off-diagonal time dependent elements of $\hat{\rho}(t)$ suitably. Our inference agrees with the conventional answer of statistical mechanics, if the possible macroscopic oscillation in $\operatorname{Tr} \hat{\rho}(t) \Delta \hat{O}_{\tau}(\vec{x})$ diminishes soon.

To analyze the effects of time averaging, one may consider a simpler example of the average position of particles (instead of the particle number density)

$$
\hat{O}=\vec{X}=\sum_{i} \vec{x}_{i} / N
$$

of the gas confined into the left-half of the box at $t=0$, which is one of the indicators of the macroscopic motion of particles. One may make a crude estimate of the typical frequency contained in $\vec{x}_{i}$ by considering the matrix element $\left\langle n+1\left|\vec{x}_{i}\right| n\right\rangle$ for a free particle as

$$
\omega \sim(\hbar \pi(n+1))^{2} /\left(2 m L^{2} \hbar\right)-(\hbar \pi n)^{2} /\left(2 m L^{2} \hbar\right) \sim(\hbar \pi n) /\left(m L^{2}\right) \sim v / L
$$

where $v=(\hbar \pi n) / m L$ is the typical velocity, which is determined by the temperature appearing in the diagonal elements $p_{n}$. Here $L$ is the size of the box. It is then unlikely that $\langle\hat{O}(t)\rangle$ contains the sizable components with frequency

$$
\omega=\left|E_{n}-E_{m}\right| / \hbar \leq 2 \pi / \Delta t_{c}
$$

to survive the time averaging for $\Delta t_{c} \gg L / v$ (or equivalently, $2 \pi / \Delta t_{c} \ll 2 \pi v / L$ ), except for the static diagonal elements. For this choice of $\hat{O}$ and the crude estimate, the gas which was in thermal equilibrium and confined into the left-half of the box at $t=0$ satisfies the condition (4.2) for $\Delta t_{c} \gg L / v$. The relaxation time in the present example is determined by the typical transport time scale $\tau \sim L / v$, provided that a soliton-like persistent collective motion does not occur. 


\subsection{Entropy law of Clausius}

The physical entropy of the final thermodynamic state defined by this time averaging (4.2), which is characterized by $\Delta t_{c}$, is estimated by

$$
\bar{H}\left(\Delta t_{c}\right) \equiv-k \operatorname{Tr} \overline{\hat{\rho}} \ln \overline{\hat{\rho}}
$$

with

$$
\overline{\hat{\rho}}=\left(1 / \Delta t_{c}\right) \int_{t}^{t+\Delta t_{c}} \hat{\rho}(t) d t
$$

for a generic value of $t$. This value is expected to be close to the maximum of Shannon's statistical amount of uncertainty $S$, which is the maximum value of any sensible definition of entropy because of (3.8): Note that

$$
S \geq \bar{H}\left(\Delta t_{c}\right)
$$

since $\overline{\hat{\rho}}_{n n}=\hat{\rho}(t)_{n n}=p_{n}$ and $\operatorname{Tr} \overline{\hat{\rho}}=1$, which are sufficient to prove (3.8). We interprete this approach of $\bar{H}\left(\Delta t_{c}\right)$ to $S$ as a manifestation of the entropy law in the present formulation of quantum statistical mechanics. ${ }^{29)}$ We reiterate that we used two ingredients to formulate the entropy law in our approach : The first is the statistical input related to the least biased inference on the basis of a limited amount of information available (3.5), and the second is the dynamical input related to the time averaging in (3.9) and (4.2).

The von Neumann entropy $H$ is in contrast rigidly defined by the basic dynamics, and it does not allow any arbitrary manipulation such as taking a time averaging of $\hat{\rho}(t)$. The dynamical entropy $H$ of von Neumann stays constant throughout the unitary time development of the system regardless of our time averaging procedure. In fact, the above time averaging (4.2) to define the physical thermodynamic state resolves the discrepancy of the dynamical von Neumann $H$ and the physical statistical entropy (4.11) we defined. Physically, the von Neumann entropy $H$ is sensitive to the dynamical motion of all the time scales in the system, whereas the thermodynamic entropy (4.11) is not sensitive to the motion with time scales shorter than $\sim \Delta t_{c}$; moreover, the condition (4.2) states that the macroscopic motion with time scales larger than $\sim \Delta t_{c}$ in the system is negligible.

We here note an interesting analogy of the present formulation of physical entropy with the renormalization group in field theory. The parameter $\Delta t_{c}$ (or to be precise, $\left.\hbar / \Delta t_{c}\right)$ characterizes the energy scale of the theory, and the entropy $\bar{H}\left(\Delta t_{c}\right) \equiv-k \operatorname{Tr} \overline{\hat{\rho}} \ln \overline{\hat{\rho}}$ in (4.11) corresponds to the renormalized running coupling constant. The ultra-violet limit $\Delta t_{c} \rightarrow 0$ gives rise to the von Neumann entropy $H$, which corresponds to the bare coupling constant, the fundamental quantity defined by the basic dynamics, namely, quantum mechanics in the present case. But physics is not sensitive to the bare coupling constant. The infrared limit of $\bar{H}$ for $\Delta t_{c} \rightarrow$ large gives rise to the measurable quantity, the maximum of Shannon's $S$, corresponding to the coupling constant $\alpha=1 / 137$ in QED defined in the Thomson limit. In this analogy, the entropy law of Clausius corresponds to a statement of the existence of a stable infrared fixed point for finite $\Delta t_{c}$.

This picture also suggests that the dynamical density matrix $\hat{\rho}(t)$ does not approach the static diagonal form in (3.6) even in thermal equilibrium, since the oscillations with

microscopic time scales always exist. The microscopic time dependence of the equilibrium 
density matrix is also expected in the conventional Gibbs ensemble theory, if one defines the thermal equilibrium by macroscopic observables $\hat{O}_{\tau}$ with well defined time scale $\tau$. Our definition of the macroscopic observables $\hat{O}_{\tau}$ in (3.10) shows that the macroscopic observables are not sensitive to the possible microscopic time (shorter than $\tau$ ) dependence of the equilibrium density matrix.

Our picture is expected to have an implication on the linear response theory for time dependent current correlations ${ }^{30}$ ) with frequencies which are comparable to $\sim 1 / \tau$, since the possible time dependence of the equilibrium density matrix is not ignored for such a case. In the applications of the fluctuation-dissipation theorem, one usually examines the correlation functions of operators averaged with the static diagonal equilibrium density matrix $e^{-\beta \hat{\mathcal{H}}} / Z$. The possible microscopic time dependence of equilibrium density matrix may lead to interesting implications on the analysis of the foundations of linear response theory.

\section{Discussion}

The purpose of this note has been to discuss quantum statistical mechanics which incorporates Shannon's statistical inference, and to analyze a resulting picture for the second law. Our statistical inference does not resolve the basic issue why the statistical inference works for a many particle system, but our statistical inference ,unlike the equal a priori probabilities, allows an analysis of the dynamical aspects of the second law.

Our picture for the general tendency toward thermal equilibrium is quite different from the one in the conventional formulation of quantum mechanical H-theorem: ${ }^{8,9,18)}$ We note that the conventional coarse-grained approach performs not only the statistical operation by setting the diagonal elements in each subsector of the density matrix to be equal by assuming equal a priori probabilities, but also the dynamical operation by setting the off-diagonal time dependent elements to be zero to let the entropy increase.

We now briefly comment on the two ambitious approaches to the second law on the basis of purely dynamical considerations, to be compared with our more conservative statistical analysis. The first is the approach of Tasaki ${ }^{31)}$ and the second is the approach of Van Hove: ${ }^{32}$

Tasaki analyzed a possible quantum mechanical derivation of a canonical ensemble starting from a pure quantum state. The basic idea is to consider two quantum systems, a bath $|\alpha\rangle$ and a subsystem $|a\rangle$, and one then introduces a suitable interaction between them so that one obtains a pure quantum state for the combined system,

$$
|\psi\rangle=\sum_{a, \alpha} M_{\alpha, a}|\alpha\rangle \otimes|a\rangle
$$

which has a vanishing von Neumann entropy. One then traces out the bath system in the density matrix

$$
\hat{\rho}_{\text {tot }}=|\psi\rangle\left\langle\psi\left|\rightarrow \hat{\rho}_{\text {sub }}=\sum_{a, b}\left(M^{\dagger} M\right)_{a b}\right| b\right\rangle\langle a|
$$


Because of the quantum entanglement of two systems, one then obtains a mixed state for the subsystem which has a non-vanishing entanglement entropy ${ }^{33)}$

$$
H_{\text {sub }}=-k \operatorname{Tr} \hat{\rho}_{\text {sub }} \ln \hat{\rho}_{\text {sub }}>0 .
$$

To reproduce a canonical ensemble for the subsystem, Tasaki assumes the "hypothesis of equal weights for eigenstates" for the combined system. He also argues the cancellation of oscillating non-diagonal components of the density matrix for the subsystem at sufficiently large $t$ by using Chebyshev's inequality. ${ }^{31)}$ If one can show that the "hypothesis of equal weights for eigenstates" holds for a rather general class of dynamical systems, the quantum entanglement entropy would provide a physical explanation of the statistical entropy. At this moment, it appears that the generality of the hypothesis has not been established.

In the analysis of entanglement entropy, a clear distinction between the von Neumann's $H$, which is equal for both of the bath and the subsystem ${ }^{33)} H_{b a t h}=H_{\text {sub }}$ and thus not extensive, and the Shannon's $S$, which could be vastly different for the bath and the subsystem and thus could be extensive, is expected to be essential.

Van Hove ${ }^{32)}$ analyzed the possible approach of a general mixed state to microcanonical states by a dynamical time development in the limit $t \rightarrow \infty$. To be specific, he starts with a mixed state

$$
\hat{\rho}(0) \equiv \int d \alpha|\alpha\rangle\left|c_{\alpha}\right|^{2}\langle\alpha|
$$

where $\hat{H}_{0}|\alpha\rangle=\epsilon_{\alpha}|\alpha\rangle$, and the unitary time development generated by

$$
U(t)=\exp \left[-i\left(\hat{H}_{0}+\lambda \hat{V}\right) t / \hbar\right] .
$$

His major claim is ( see Eq. (1.4) in the first paper in ref. 32)

$$
\operatorname{Tr} \hat{O} U(t) \hat{\rho}(0) U^{-1}(t) \rightarrow\langle\hat{O}\rangle_{\text {microcanonical }}
$$

for $t \rightarrow \infty$. Since the operator $U(t)$ is unitary for whatever large but finite $t$, both of the von Neumann's $H$ and Shannon's $S$ remain at the initial value different from the microcanonical value. Van Hove however considers a singular limit $\lambda^{2} t \rightarrow$ constant for $t \rightarrow \infty$ (i.e., $\lambda t \sim 1 / \lambda \rightarrow \infty$ for $t \rightarrow \infty$ ). In such a limit, if literally taken, the $S$-matrix

$$
\hat{S}=\lim _{t_{ \pm} \rightarrow \pm \infty} e^{i \hat{H}_{0} t_{+} / \hbar} e^{-i\left(\hat{H}_{0}+\lambda \hat{V}\right)\left(t_{+}-t_{-}\right) / \hbar} e^{-i \hat{H}_{0} t_{-} / \hbar}
$$

(and consequently perturbation theory he uses) is not defined, since the condition of an adiabatic switch-on and switch-off of the interaction is not satisfied. See also ref. 34. If one can provide a mathematical basis for the singular limit, one would be able to derive the microcanonical ensemble from a general mixed state by a unitary time development. At this moment, to our knowledge, such a mathematical basis appears to be missing.

Finally, we mention recent activities on an alternative approach to the second law. Jarzynski ${ }^{35)}$ found the following amusing identity

$$
\begin{aligned}
\frac{Z_{1}}{Z_{0}} & =\frac{1}{Z_{0}} \int d \mu\left(z^{\prime}\right) e^{-\beta H_{1}\left(z^{\prime}\right)} \\
& =\frac{1}{Z_{0}} \int d \mu\left(z^{\prime}\right) e^{-\beta H_{0}(z)-\beta\left(H_{1}\left(z^{\prime}\right)-H_{0}(z)\right)} \\
& =\frac{1}{Z_{0}} \int d \mu(z) e^{-\beta H_{0}(z)-\beta W(z)}=\left\langle e^{-\beta W(z)}\right\rangle
\end{aligned}
$$


where we defined the mapping

$$
z=\left(q\left(t_{0}\right), p\left(t_{0}\right)\right) \rightarrow z^{\prime}=\left(q^{\prime}\left(t_{1}\right), p^{\prime}\left(t_{1}\right)\right)
$$

as the canonical transformation generated by the time dependent Hamiltonian (a quantum version is also known ${ }^{36)}$ )

$$
H_{\lambda(t)}, \quad t_{0} \leq t \leq t_{1}
$$

with

$$
\lambda\left(t_{0}\right)=0, \quad \lambda\left(t_{1}\right)=1 .
$$

The Liouville theorem $d \mu\left(z^{\prime}\right)=d \mu(z)$ is essential in the above identity. We also defined the work done during the time development by $W(z) \equiv H_{1}\left(z^{\prime}\right)-H_{0}(z)$.

If one defines the Helmholtz free energy for the system described by Hamiltonian $H_{1}$ at temperature $\beta$ by $F_{1}(\beta)$, one obtains from (5.8)

$$
\Delta F(\beta)=F_{1}(\beta)-F_{0}(\beta)=-\frac{1}{\beta} \ln \left\langle e^{-\beta W(z)}\right\rangle .
$$

and, by noting the mathematical inequality $\langle\exp [A(z)]\rangle \geq \exp [\langle A(z)\rangle]$,

$$
\Delta F(\beta) \leq\langle W\rangle
$$

which resembles the basic thermodynamic inequality, ${ }^{6)}$ an alternative expression of the second law. The identity (5.8) as it stands is equivalent to the Liouville theorem and thus contains no information about the thermal entropy generation. In fact, it is known (from an explicit analysis of harmonic oscillators, for example) that the equality sign in (5.13) does not hold for an infinitely slow adiabatic work ${ }^{35)}$, which is by itself consistent: But to analyze the equality sign in (5.13), one need to analyze the approach of a system in (5.8), once driven out of equilibrium by an external work, to thermal equilibrium again. The analysis of Jarzynski is thus complementary to the analysis in the present note, and certainly it does not replace our analysis. See recent works ${ }^{37,38}$ related to the above identity. See also Lenard ${ }^{39)}$ for an analysis of similar inequality associated with a canonical ensemble. ${ }^{40)}$

In conclusion, we have illustrated a physical picture of the second law when one makes a clear distinction between statistical aspects and dynamical aspects in statistical mechanics, which is made possible if one uses $S$ in (3.2). Our basic view as presented here is rather conservative, namely, it is based on the premise that the entropy law of Clausius is not a direct consequence of microscopic dynamical laws alone. In the context of classical Boltzmann approach, this view appears to be shared with experts just to quote " It follows that the macroscopic dynamics cannot be a consequence of the microscopic dynamics alone". ${ }^{10)}$ The remaining basic issue in the present approach is to specify precisely the class of many-particle Hamiltonians which ensure (4.2).

I thank H. Tasaki for numerous clarifying comments, and A. Shimizu and M. Ueda for helpful comments at the initial stage of this work. 


\section{References}

[1] C. E. Shannon: Bell System Tech. J. 27(1948) 379; ibid. 623.

[2] E. T. Jaynes: Phys. Rev. 106(1957) 620.

[3] L. D. Landau: Z. Physik 45(1927) 430.

[4] J. von Neumann: Goettinger Nachr.1(1927) 273; Collected Works, ed. A. H. Taub (Pergamon Press, Oxford, 1961) Vol.1, p.208.

[5] E. T. Jaynes: Phys. Rev. 108(1957) 171.

[6] L. D. Landau and E. M. Lifshitz: Statistical Physics (Pergamon Press, Oxford, 1985) 3rd ed.

[7] A. Isihara: Statistical Physics (Academic Press, New York, 1971).

[8] D. ter Haar: Elements of Thermostatistics (Holt, Reinhart and Winston, New York, 1966) 2nd ed., and references therein.

[9] D.N. Zubarev: Nonequilibrium Statistical Thermodynamics (Consultants Bureau, New York, 1974), and references therein.

[10] O.E. Lanford: Physica 106A(1981) 70.

[11] J. L. Lebowitz: Physica 194A(1993) 1, and references therein.

[12] J. Bricmont: Science of Chaos or Chaos in Science?, chao-dyn/9603009.

[13] There appears an important difference between $H$ and $S$ if one allows a time dependent Hamiltonian. The density matrix is still transformed by a unitary transformation and thus $H$ stays constant. But $S$ generally changes except for an infinitely slow adiabatic variation of the Hamiltonian.

[14] J. G. Kirkwood: J. Chem. Phys. 14(1946) 180; ibid. 15 (1947)72.

N. N. Bogolyubov: Studies in Stat. Mech. 1(1962) 1.

E. D. G. Cohen and T. H. Berlin: Physica 26(1960) 717.

[15] One can confirm the relation $\frac{d S}{d E}=k \beta(E)$ for this solution, and we assume $\beta(E)>0$.

[16] One may start with normalized pure states in the energy representation $\left|\Psi_{k}\right\rangle=$ $\sum_{n} a_{k, n}|n\rangle$, and one may define a density matrix for a mixed state by $\hat{\rho}=$ $\sum_{k} b_{k}\left|\Psi_{k}\right\rangle\left\langle\Psi_{k}\left|=\sum_{k} \sum_{m} \sum_{n} b_{k} a_{k, m} a_{k, n}^{\star}\right| m\right\rangle\left\langle n\left|\equiv \sum_{m, n} \rho_{m, n}\right| m\right\rangle\langle n|$ with $b_{k}>0$ and $\sum_{k} b_{k}=1$. It is however common to define a mixed state by using more general $\rho_{m, n}$, which we adopt in the present note.

[17] This statistical inference of diagonal elements means that the macroscopic observables $\langle E\rangle$ and $\langle N\rangle$ are insensitive to whether one uses the original density matrix or the density matrix after one applied the statistical inference. 
[18] W. Pauli: Probleme der Modernen Physik, Sommerfeld Festschrift, ed. P. Debye (Hirzel, Leipzig, 1928);

R. C. Tolman: The Principles of Statistical Mechanics (Oxford University Press, New York, 1938).

[19] A. Wehrl: Rev. Mod. Phys. 50 (1978) 221.

[20] A sketch of the proof is as follows: Define $f(x)=-k x \ln x$ for $0 \leq x \leq 1$, which is convex in the sense $f^{\prime \prime}(x)<0$ for $0<x \leq 1$. Define any orthonormal set of states $\{|n\rangle\}$ in terms of the normalized eigenstates $\varphi_{k}$ of $\hat{\rho}$ as, $|n\rangle=\sum_{k} a(n)_{k} \varphi_{k}, \quad \sum_{k} a(n)_{k} a(l)_{k}^{\star}=$ $\delta_{n l}, \quad \hat{\rho} \varphi_{k}=\lambda_{k} \varphi_{k}, \quad \lambda_{k} \geq 0, \quad \sum_{k} \lambda_{k}=1$. If one defines a tangential line to $f(x)$ at $x=\langle n|\hat{\rho}| n\rangle=\sum_{k}\left|a(n)_{k}\right|^{2} \lambda_{k}$ by $\bar{f}(x)=a x+b$, one has $f\left(\lambda_{k}\right) \leq \bar{f}\left(\lambda_{k}\right)$ and $\langle n|\bar{f}(\hat{\rho})| n\rangle=\sum_{k}\left|a(n)_{k}\right|^{2} \bar{f}\left(\lambda_{k}\right)=\bar{f}(\langle n|\hat{\rho}| n\rangle)=f(\langle n|\hat{\rho}| n\rangle)$. One can then prove ${ }^{21)}$ $\langle n|f(\hat{\rho})| n\rangle=\sum_{k}\left|a(n)_{k}\right|^{2} f\left(\lambda_{k}\right) \leq \bar{f}(\langle n|\hat{\rho}| n\rangle)=f(\langle n|\hat{\rho}| n\rangle)$, where the equality sign holds only for $\hat{\rho}|n\rangle=\lambda_{n}|n\rangle$.

One thus concludes $\sum_{n}\langle n|f(\hat{\rho})| n\rangle=-k \operatorname{Tr} \hat{\rho} \ln \hat{\rho} \leq \sum_{n} f(\langle n|\hat{\rho}| n\rangle)=$ $-k \sum_{n}\langle n|\hat{\rho}| n\rangle \ln \langle n|\hat{\rho}| n\rangle$, as required.

[21] R. Peierls: Phys. Rev. 54(1938) 918.

[22] P. A. M. Dirac: Principles of Quantum Mechanics (Oxford University Press, Oxford, 1958).

[23] Intuitively, one can see a possible origin of the violation of time reversal symmetry in this time averaging. One cannot reproduce the original density matrix form the time averaged one, and in this sense the use of macroscopic observables induces the violation of microscopic time reversal invariance.

[24] To be more precise, the intrinsic time scale $\tau$ is defined as follows: For a given operator $\hat{O}$, one first defines the maximum value of $\tau$ which satisfies (3.10) for each $\hat{\rho}$. Based on the set of those values $\{\tau(\rho)\}$, one may define the intrinsic time scale associated with $\hat{O}$ by the minimum of $\tau(\rho)$, namely, $\tau=\min _{\rho}\{\tau(\rho)\}$. In practice, however, the time dependence of an observable always appears in the combination $\operatorname{Tr} \hat{\rho}(t) \hat{O}$ in our treatment. One may thus use the time scale given by the maximum of $\tau$ which satisfies (3.10) for a given physical system specified by $\hat{\rho}(t)$.

[25] P. Bocchieri and A. Loinger: Phys. Rev. 107(1957) 337.

I.C. Percival: J. Math. Phys. 2(1961) 235.

[26] One may avoid the recurrence theorem by appealing to the thermodynamic limit with $V \rightarrow$ large and the particle density $N / V$ kept finite, but at the same time one looses the dynamical information of the system. We know very little about the dynamical properties of a many particle system, and the reccurence theorem provides precious information.

[27] For a particle number $N=10^{23}$ at room temperature, $\Delta E \sim k T \times \sqrt{N} \sim 10^{10} \mathrm{eV}$. If one chooses $\Delta t_{c} \sim 10^{-1} \mathrm{sec}$, for example, $2 \pi \hbar / \Delta t_{c} \sim 10^{-13} \mathrm{eV}$. 
[28] A similar consideration is applicable to the analysis of a time dependent Hamiltonian if one analyzes the behavior of the system after switching off the time dependent perturbation.

[29] This picture suggests that the bulk of entropy generation takes place within the time scale of relaxation measured by macroscopic observables.

[30] H. B. Callen and T. A. Welton: Phys. Rev. 83(1951) 34;

R. Kubo: J. Phys. Soc. Jpn. 12(1957) 570;

H. Nakano: Int. J. Mod. Phys. B7(1993) 2397 and references therein.

[31] H. Tasaki: Phys. Rev. Lett. 80(1998) 1373.

[32] L. Van Hove: Physica 25(1959) 268; idid. 21(1955) 517.

[33] H. Araki and E. H. Lieb: Comm. Math. Phys. 18 (1970)160;

L. Bombelli, R. K. Koul, J. Lee, and R. D. Sorkin: Phys. Rev. D34 (1986) 373;

M. Srednicki: Phys. Rev. Lett. 71(1993)666;

H. Terashima: Phys. Rev. D61(2000)104016, and references therein.

[34] R. Ingraham: Nuovo Cim. 9 (1958) 99.

[35] C. Jarzynski: Phys. Rev. Lett. 78 (1997) 2690; Phys. Rev. E56 (1997) 5018; J. Stat. Phys. 96 (1999) 415.

[36] S. Yukawa: J. Phys. Soc. Jpn, 69 (2000) 2367.

[37] G.E. Crooks: J. Stat. Phys. 90 (1998) 1481, and references therein.

[38] H. Tasaki:The second law of thermodynamics as a theorem in quantum mechanics, cond-mat/0011321, and references therein.

[39] A. Lenard: J. Stat. Phys. 19 (1978) 575.

[40] The essence of the inequality in ref. 39 is stated in our framework as follows: One may denote the density matrix for a (micro-)canonical ensemble (3.6) by $\hat{\rho}_{0}$ and the corresponding average energy by $E_{0}$. After an arbitrary small unitary transformation, we have $\hat{\rho}=U \hat{\rho}_{0} U^{\dagger}$ and $E=\sum_{n} E_{n}^{\prime}\langle n|\hat{\rho}| n\rangle$. Our inequality (3.8) indicates $S=$ $-k \sum_{n}\langle n|\hat{\rho}| n\rangle \ln \langle n|\hat{\rho}| n\rangle \geq H=-k \operatorname{Tr} \hat{\rho} \ln \hat{\rho}=-k \operatorname{Tr} \hat{\rho}_{0} \ln \hat{\rho}_{0}=S\left(E_{0}\right)$. The maximum of $S$ with fixed $E$, which is the physical entropy if relaxation takes place, thus satisfies $S(E) \geq S\left(E_{0}\right)$. The condition $d S\left(E_{0}\right) / d E_{0}=k \beta\left(E_{0}\right)>0$ then implies $E \geq E_{0}$ for an arbitrary small unitary transformation $U$, which includes time development generated by a time-dependent Hamiltonian. ${ }^{39)}$ Our analysis in this paper is complementary to that of ref. 39 where no analysis of relaxation after an external perturbation is given. 